\title{
EFFECT OF ASPIRIN IN THE KIDNEY OF WISTAR ALBINO RATS- A HISTOMORPHOLOGICAL STUDY
}

\author{
Awasthi JR ${ }^{* 1}$, Pandey $\mathrm{N}^{2}$, Yadav $\mathrm{G}^{2}$, Shrestha $\mathrm{S}^{2}$, Sarraf $D \mathrm{P}^{3}$, Jha $C \mathrm{CB}^{2}$ \\ ${ }^{* 1}$ Department of Human anatomy, Gandaki Medical College, Pokhara, Nepal \\ ${ }^{2}$ Department of Human anatomy, BP Koirala Institute of Health Sciences, Dharan, Nepal \\ ${ }^{3}$ Department of Clinical Pharmacology and Therapeutics, BP Koirala Institute of Health Sciences, Dharan, Nepal
}

\section{ABSTRACT}

Background: Aspirin is the acetylated form of the Salicylic acid. Salicylic acid has been used since ancient times to provide pain relief and treat inflammatory conditions. It is a phenolic compound present in plants, where it plays a central role in the development of local and systemic resistance to pathogen infection. Humans and animals obtain SA mainly from daily foods, fruits, and vegetables.

Objectives: To determine the effect of aspirin in the kidney of Wistar albino rat.

Materials and Methods: Sixty rats were randomly divided into equal two groups. Group I (control) was injected (i.p.) $1 \mathrm{ml}$ of saline per day. Group II was given $100 \mathrm{mg} / \mathrm{kg}$ aspirin daily i.p. All groups were treated over a period of eight consecutive days and on $9^{\text {th }}$ day; rats were sacrificed. Kidney slides were prepared microscopic examination. Independent t-test was used for data analysis.

Results: Examination of renal cortex from the both control group and aspirin treated rats showed normal parenchyma. However, in the latter group only few tubules showed the loss of brush border and necrosis but they were not desquamated. The oculometric analysis of renal tubules showed that diameter of the proximal and distal convoluted tubules, and renal corpuscles were similar in both the group. However, the diameter of glomerulus was significantly increased and the renal space was significantly reduced in aspirin treated rats compared to normal.

Conclusion: Thus, aspirin shows minimal renal toxic effect, however, all the histo-architecture of the kidney was normal.

Keywords: Aspirin, Nephrotoxicity, Kidney, Wistar albino rats

\section{INTRODUCTION}

Aspirin is the acetylated form of the salicylic acid. Salicylic acid (SA) has been used since ancient times to provide pain relief and treat inflammatory conditions. Salicylic acid is a phenolic compound present in plants, where it plays a central role in the development of local and systemic resistance to pathogen infection.(1) Humans and animals obtain SA mainly from daily foods, fruits, and vegetables.

Increasing evidence demonstrates that applied SA can counteract oxidative damage induced by adverse conditions in animals(2) though the mechanisms underlying these effects remain unclear.(3) It has been reported that SA comprise free radical-scavenging and iron chelation properties.(3) Studies have shown that salicylate effectively protects against gentamicin induced

*Corresponding author:

Email: janu.awasthi@gmail.com

http://dx.doi.org/10.20530/IJTA 33 135-138

ISSN 2320-138X (c) 2016 hearing loss in guinea pigs.(4)'(5) and against its nephrotoxic effect in Wistar albino rats.(6)

The aim of this study was to investigate whether aspirin (acetylated salicylic acid) treatment is safe to the kidney or not. The positive result will be helpful in suggesting the aspirin as adjunct medication in drugs therapy having oxidizing damaging effect e.g. with gentamicin. The analysis of the drug effect was done through the examination \& evaluation of histopathological effects of rat's kidney.

\section{MATERIALS AND METHODS}

\subsection{Animals:}

In this experimental study; sixty healthy Wistar albino rats of either sex weighing 100-200 gram were randomly selected. The rats were acclimatized for four weeks before enrolling in the study. The animals were housed in each plastic cages made up of propylene with husk bed and were placed in room at atmospheric temperature and humidity with 12hour light-dark cycles without any stressful stimuli. The rats were provided free access to standard rodent diet and water ad libitum. All experimental 
procedures were conducted in accord with ethical guidelines of Nepal Health Research Council (NHRC) for the care and use of animals in health research in Nepal (7) and approval was taken from the institutional ethical review board, BP Koirala Institute of Health Sciences, Nepal, before conducting the study. All efforts were made to minimize animal suffering and reduce the number of animals used.

\subsection{Drugs:}

Aspirin (USV limited, India) was bought from local market and the injecting solution was prepared by dissolving it in normal saline.

\subsection{Experimental Protocol:}

After a quarantine period, sixty rats were randomly divided into two groups, each consisting of thirty animals. Group I was used as control and was injected $1 \mathrm{~mL}$ of saline intraperitoneally (i.p.) per day. Group II was injected only aspirin in single dose of $100 \mathrm{mg} / \mathrm{kg}$ body weight i.p. daily. All groups were treated over a period of eight consecutive days. Twenty-four hours after the administration of last doses, on 9th day, rats were sacrificed by cervical dislocation. Kidneys were taken out by abdominal dissection, weighed and preserved in $10 \%$ buffered formaldehyde solution. The preserved kidneys were processed and sectioned using rotary microtome. The sections were then stained by using Harris's hematoxylin and Eosin Y.

\subsection{Histopathological Examinations:}

After the slide preparation, blind observations were made using the light microscope. For each rat; 100 PCT and DCT associated with glomeruli were examined from randomly selected minimum of 8 fields for each kidney section. Ocular micrometer was used for quantitative measurements of diameter of renal corpuscles, glomerulus, PCT, DCT, and dimension of Bowman's space.

\subsection{Statistical Analysis:}

Statistical analysis was performed by the use of SPSS software version 20 (IBM corp.). Results were presented as mean value $\pm S D$. The independent $t$-test test was used for comparisons of diameter of tubules, renal corpuscles, glomerulus, renal space of the control and test groups. Probability values $(P)$ less than 0.05 was considered statistically significant.

\section{RESULTS}

All animals of control group (I) and aspirin
Table 1: Mean proximal convoluted tubule diameter (PCT) of different groups

\begin{tabular}{lc}
\hline Group $(\mathbf{n = 3 0 0 0 )}$ & $\begin{array}{c}\text { PCT diameter } \\
(\boldsymbol{\mu m})\end{array}$ \\
\hline Normal (I) & $35.52 \pm 4.05$ \\
Aspirin treated (II) & $35.15 \pm 2.67$ \\
$P$ value & $>0.05$ \\
\hline
\end{tabular}

Table 2: Mean distal convoluted diameter (DCT) diameter of different groups

\begin{tabular}{lc}
\hline Group $(\mathbf{n = 3 0 0 0 )}$ & $\begin{array}{c}\text { DCT diameter } \\
(\boldsymbol{\mu m})\end{array}$ \\
Normal (I) & $32.60 \pm 4.25$ \\
Aspirin treated (II) & $32.14 \pm 2.28$ \\
$P$ value & $>0.05$ \\
\hline
\end{tabular}

Table 3: Mean renal corpuscle diameter of different groups

\begin{tabular}{lc}
\hline Group ( $\mathbf{n = 3 0 0 0 )}$ & $\begin{array}{c}\text { Renal corpuscle } \\
\text { diameter }(\mu \mathrm{m}) \\
\text { (mean } \pm \text { SD) }\end{array}$ \\
Normal (I) & $83.68 \pm 8.17$ \\
Aspirin treated (II) & $84.40 \pm 5.63$ \\
$P$ value & $>0.05$ \\
\hline Table 4: Mean & glomerular diameter of \\
different groups & glomerulus diameter \\
\hline Group ( $\mathbf{n}=\mathbf{3 0 0 0 )}$ & $(\boldsymbol{\mu m})$ \\
\multicolumn{2}{c}{ (mean \pm SD) } \\
\hline Normal (I) & $74.74 \pm 8.19$ \\
Aspirin treated (II) & $77.08 \pm 6.51$ \\
$P$ value & $<0.001$ \\
\hline
\end{tabular}

Table 5: Mean renal space of different groups

\begin{tabular}{lc}
\hline Group $(\mathbf{n = 3 0 0 0 )}$ & $\begin{array}{c}\text { Renal space }(\boldsymbol{\mu m}) \\
(\mathbf{m e a n} \pm \text { SD) }\end{array}$ \\
\hline Normal (I) & $5.06 \pm 1.65$ \\
Aspirin treated (II) & $4.04 \pm 1.95$ \\
$P$ value & $<0.001$ \\
\hline
\end{tabular}

treated group (II) were healthy and active. Examination of renal cortex from group I (Control) showed normal renal corpuscles. The cortical tubules made the bulk of parenchyma and mainly consisted 
of proximal (PCT) and distal convoluted tubules (DCT). PCT were lined by simple cuboidal epithelium, having prominent brush borders and acidophilic cytoplasm. DCT were identified on account of simple cuboidal epithelium, clearly defined and wider lumen and closely packed nuclei per section (Figure$1)$.

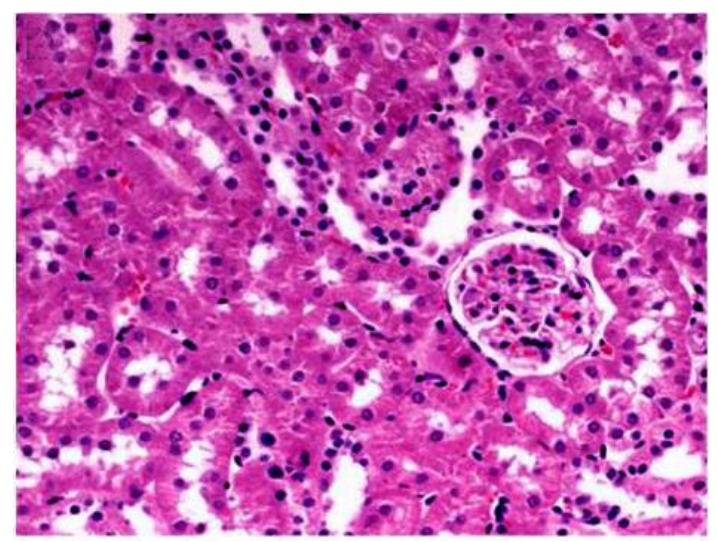

Figure 1: Photomicrograph of rat kidney section showing normal histology in control group [X400]

Examination of the renal cortex of aspirin treated rats (group II) showed almost normal architecture of renal corpuscles and cortical tubules. Only few tubules showed the loss of brush border and necrosis but they were not desquamated (Figure-2). It signifies that aspirin was found to be safe as it did not induce appreciable histopathological changes in the kidney.

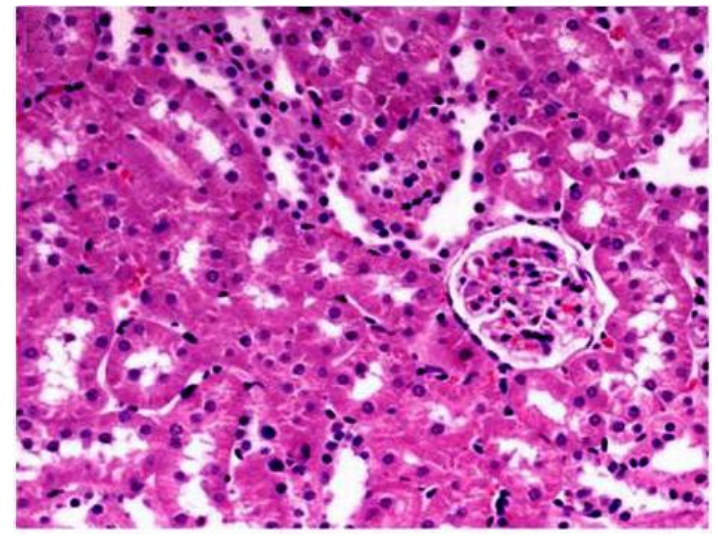

Figure 2: Photomicrograph of rat kidney section showing almost normal histology in aspirin treated group [X400]. Glomerulus is slightly enlarged and renal space is slightly reduced compared to normal group.

One PCT and DCT lying adjacent to each renal corpuscle were selected blindly from different randomly chosen fields of renal cortex. Thus, total 3000 renal corpuscle ( $\mathrm{rc}$ ), 3000 glomerulus (glo), 3000 PCT and 3000 DCT (30 rats per group $\times 100$ PCT, $\mathrm{DCT}, \mathrm{rc}$, glo per rat) in a group were measured and average was taken. The data of the measurement of PCT, DCT, renal corpuscle, and glomerulus are summarized in Table-1, 2, 3.

Independent t-test showed that there was no significant difference between the diameter of PCT, DCT and renal corpuscle of group I vs II, suggesting that aspirin causes no histologically harmful effect on these structures of Kidney. Similarly, diameter of 3000 glomerulus and dimension of renal space were measured and average was taken. The data of these measurements are summarized in Table- 4 and 5 . Independent t-test showed that there was significant difference between the diameter of glomerulus and dimensions of renal space of group I vs II. It suggests that of use aspirin leads the significant increase in diameter of glomerulus and decrease in renal space. Moreover, it implies that aspirin is minimally toxic to renal structures.

Therefore, in this study; light microscopic examination of kidneys from control and aspirin treated rats showed almost no structural alterations in renal tissues. Study by Randjelovic et al. showed considerably mild glomerular and tubular damage in rats treated with both Gentamicin and salicylic acid (SA), thus showing nephron protective effect of SA. It suggests that aspirin alone is not harmful to kidney. The same result is verified by our study as well, however it has some dilating effect on glomerulus leading to congestion of renal space.

\section{CONCLUSION}

Result of this study suggested that aspirin is not harmful to the kidney. However, it causes small changes (decrease) in the renal space and increase in the diameter of glomerulus. It needs further molecular level study to see whether aspirin has nephrotoxic effect or not.

\section{Competing interests}

Authors have no conflict of interest. The study was conducted as the partial fulfillment of the requirement of degree of masters in science in Human Anatomy of BP Koirala Institute of Health Sciences, Dharan, Nepal

\section{Authors' Contribution}

JRA was the principal investigator of this research and has the main role from experiment to 
manuscript preparation. NP, GY participated in the design of the study and manuscript drafting. SS and CBJ were involved in analysis and interpretation of the microscopic observation and in drafting the manuscript. All authors read and approved the final manuscript.

\section{ACKNOWLEDGEMENT}

I would like to acknowledge the role of Dr. Deependra Prasad Sarraf who helped in proof reading the pharmacological aspect of the study. I am thankful to Mr. Shailesh Adhikari and all the staffs of the Department of Anatomy for their continuous support in course of the study.

\section{REFERENCES}

1. DEMPSEY D, KLESSIG D. Salicylic acid, active oxygen species and systemic acquired resistance in plants. Trends in Cell Biology. 1994 Sep;4(9):334-8. Available from: http://dx.doi.org/10.1016/09628924(94)90235-6.

2. Guerrero A, González-Correa J., Arrebola M., MuñozMarın J, Sánchez De La Cuesta F, De La Cruz J. Antioxidant effects of a single dose of acetylsalicylic acid and salicylic acid in rat brain slices subjected to oxygen-glucose deprivation in relation with its antiplatelet effect. Neuroscience Letters. 2004 Apr;358(3):153-6. Available from: http://dx.doi.org/10.1016/j.neulet.2004.01.036.

3. Aruoma OI, Halliwell B. The iron-binding and hydroxyl radical scavenging action of anti-inflammatory drugs. Xenobiotica. 1988 Jan;18(4):459-70. Available from: http://dx.doi.org/10.3109/00498258809041682.

4. Sha SH, Schacht J. Salicylate attenuates gentamicininduced ototoxicity. Lab Invest. 1999 Jul;79(7):80713.

5. Chen Y, Huang W-G, Zha D-J, Qiu J-H, Wang J-L, Sha S$\mathrm{H}$, et al. Aspirin attenuates gentamicin ototoxicity: From the laboratory to the clinic. Hearing Research. 2007 Apr;226(1-2):178-82. Available from: http://dx.doi.org/10.1016/j.heares.2006.05.008.

6. Randjelovic P, Veljkovic S, Stojiljkovic N, JankovicVelickovic L, Sokolovic D, Stoiljkovic M, et al. Salicylic Acid Attenuates Gentamicin-Induced Nephrotoxicity in Rats. The Scientific World Journal. 2012; 2012:1-6. Available from: http://dx.doi.org/10.1100/2012/390613.

7. Pahari SK, Banmali P, Thaler JL. Ethical guidelines for the care and use of animals in health research Edition : First 2005. 